\title{
Escritos para pensar lo público-político en tiempos de algoritmos y plataformas conectivas
}

\section{Writings to think the public-political in times of algorithms and connective platforms}

\section{Escritos para pensar o público-político em tempos de algoritmos e plataformas conectivas}

\section{MARÍA CECILIA REVIGLIO}

maria.reviglio@fcpolit.unr.edu.ar - Editora Invitada / Consejo de Investigaciones delaUniversidad Nacional de Rosario (UNR) y Centro de Investigaciones en Mediatizaciones(CIMUNR), Argentina.

\section{NATALIA RAIMONDO ANSELMINO}

nraimondo@conicet.gov.ar - Editora invitada / Consejo Nacional de Investigaciones Científicas y Técnicas (CONICET) y Centro de Investigaciones en Mediatizaciones (CIM-UNR), Argentina.

Las discusiones sobre lo público se han suscitado en el debate académico desde las primeras conceptualizaciones realizadas entre fines de la década del '50 y principios de los ' 60 del siglo XX por Hannah Arendt y Jürgen Habermas en sus ya clásicos La condición humana e Historia y crítica de la opinión pública, respectivamente. Los cuestionamientos, atenuaciones y respuestas a estos planteos no tardaron en aparecer, incluso, contemporáneamente a la publicación de las obras mencionadas. A aquel conjunto de planteos - que varios autores de este volumen recuperan y problematizan - se suman hoy los diferentes argumentos que se han precipitado en relación con los recientes cambios acaecidos en la configuración de la esfera pública, sobre todo aquellos vinculados con el desarrollo del proceso histórico de mediatización. 
En la actualidad, lo público se presenta con una fisonomía en la que se distinguen, cada vez con mayor definición, la presencia de las plataformas conectivas (van Dijck, 2016) y las redes sociales en Internet (RSI), que parecen ir tomando mayor protagonismo en la configuración de lo común político. En ese sentido, las sociedades actuales, altamente mediatizadas, seven atravesadas por las nuevas tensiones que dicha presencia ha inaugurado. Gran parte de la vida social, al mismo tiempo que la experiencia individual, parecen confluir en estos nuevos espacios donde lo público, lo privado y lo íntimo suelen entrar en tensión y solaparse: desde protestas sociales convocadas a través de plataformas como las de Facebook, Twitter o WhatsApp, hasta los encuentros amorosos o sexuales vía Tinder, pasando por campañas políticas diseñadas especialmente para esos entornos mediáticos, por manifestaciones de la indignación popular expresadas exclusivamente en el territorio de las RSI o, también, por la circulación de información periodística quelos propios medios tradicionales adaptan o producen para estos formatos.

Los problemas vinculados con las cuestiones de lo público político tienen ya muchas décadas de abordaje y se han ido complejizando conforme lo fueron haciendo nuestras sociedades, en estrecha relación con el desarrollo del ecosistema de medios: los medios de comunicación masiva, primero; Internet y todas sus derivaciones, después. Los medios tradicionales, por su parte, han ido sufriendo constantes mutaciones a lo largo de su historia, dando lugar a lo que Verón (2013) llamó alteraciones y/o rupturas de escala. La emergencia de ambientes donde una multiplicidad de discursos se visibilizan y circulan a escalas y velocidades inesperadas - esos "nuevos espacios de enunciación pública" (XX), como los designa en su texto Víctor Castrelo-, hace necesario repensar los supuestos, las categorías y el herramental metodológico adecuados para comprender la dinámica actual de producción de lo público.

Ese es el desafío que nos propusimos en el presente número de la revista InMediaciones de la Comunicación: compilar una serie de reflexiones e investigaciones que, desde perspectivas teóricas y metodológicas diversas, aportaran herramientas al debate sobre la configuración contemporánea de lo público político. Si bien es cierto que, en los últimos años, la producción académica referida a estos aspectos dela mediatización haido en aumento, en consonancia con la pregnancia de los mismos en la vida social, creímos oportuno reunir, en un mismo volumen, trabajos que aborden el tópico en cuestión - a todas luces complejoy, por momentos, con bordes porosos-desde múltiples miradas que hasta, pueden ser, en ocasiones, contradictorias entre sí.

El resultado de esta apuesta se materializó en un monográfico en el que se pueden reconocer cinco grupos de textos vinculados la temática que nos convoca: las mediatizaciones de lo público político en tiempos de RSI.

El primero de ellos, está inaugurado por las exquisitas reflexiones de Peter Dahlgren en torno a la creciente atención otorgada al afecto — que "implica 
el lado colectivo de la emocionalidad" (p. 33) — en relación con el campo de la participación política, es decir, con el tipo de participación que, al suponer una intervención en las relaciones de poder, involucra irremediablemente confrontación y lucha. Su texto, La participación en línea en la esfera pública. Las ambigüedades del afecto, que es un honor haber podido publicar en español, es el primero de los cuatro artículos de corte teórico con los que comienza este Volumen 13(1) de InMediaciones de la Comunicación. Sus enseñanzas conducen a la necesaria superación de la clásica dicotomía racionalidad-emocionalidad y advierten sobre la presente conformación de una zona de confort en el marco de la cual la participación, más individual que colectiva, en vez de ser una experiencia de la solidaridad, se reduce muchas veces a una "actividad realizada en privado frente a la pantalla” (p. 37). En segundo lugar, se encuentra el texto de Mariano Vázquez, Esfera pública virtual. Una lectura política y comunicacional de lo público en la web, en el que se presenta un novedoso modo de abordaje de las interacciones producidas en una multiplicidad de interfaces: la esfera pública virtual (EPV). Construida en función de estudiar las discusiones en torno al conflicto denominado "El Famatina no se toca", la EPV de Vázquez provee una nueva vía de producción de conocimiento sobre fenómenos de lo público-político que no sólo involucra discursos mediatizados sino, también, abrela posibilidad de integrar las prácticas que tienen lugar en el territorio tanto online como offline. En tercer lugar, el artículo La esfera pública habermasiana. Su obsolescencia en tiempos de nuevas plataformas digitales, de Víctor Castrelo, nos propone una revisión crítica y pormenorizada dela clásica noción de esfera pública, poniéndola en tensión en el marco del estadio actual de la mediatización. Tal como también lo señala el texto de Vázquez, pensar hoy la esfera pública supone advertir, según Castrelo, la imposibilidad de seguir confinándola al terreno de las relaciones interpersonales espacialmente situadas y desprovistas de toda polémica. El autor propone, entonces, una mirada ampliada de la misma que esté centrada no ya en relaciones binarias sino triádicas. Por su parte, el cuarto lugar del primer subgrupo de escritos es ocupado por el texto denominado Un complejo salvaje. Persistencias del espacio público en la era de las redes sociales. Allí, Mariano Fernández pone el acento en el carácter cada vez más complejo del espacio público, cavilando en torno a un espacio público que nunca tuvo un estatuto estable pero que, en la actualidad, se ve particularmente tensionado por plataformas conectivas. Estos nuevos espacios, entre otras peculiaridades, posibilitan la gestación de comunicaciones interpersonales mediatizadas cuyo horizonte de destinación ya no es privado, sino público.

El segundo grupo está compuesto por tres artículos que presentan resultados de investigaciones de corte discursivo o semiolingüístico. En el primero de ellos, La argumentación denegativa del yo. El discurso de perfil en aplicaciones para encuentros íntimos, Manuel Libenson se sitúa en uno de los extremos del 
abanico presentado en el volumen —el de las experiencias interindividuales en el plano de lo íntimo amoroso- para indagar el funcionamiento argumentativo, polifónico y dialógico de la denegación en la presentación del yo en las aplicaciones Tinder y Grindr, entendidas como dispositivos discursivos. Los otros dos artículos del conjunto -Contradestinación inclusiva: el discurso de Scioli y de Macri en Facebook, de Emmanuel Pérez Zamora y \#TinelliMercenarioK. La mediatización del presidente argentino y los trolls macristas, de Ana Slimovich - abordan, en cambio, casos vinculados con lo colectivo, centrándose el primero en discursos mediatizados a través de Facebook y, el segundo, fundamentalmente de Twitter. Pérez Zamora presenta el hallazgo de un nuevo modo de contradestinación en el discurso político, la contradestinación inclusiva, resultante del análisis de los posteos que los candidatos presidenciales Mauricio Macriy Daniel Scioli publicaron en sus cuentas oficiales de Facebook en ocasión del ballotaje de 2015 en Argentina. Por su parte, Slimovich, articulando la perspectiva sociosemiótica con el dispositivo analítico de la circulación hipermediática, se adentra en las derivas discursivas - de internautas, tanto anónimos como con reconocimiento público, de periodistas y medios de comunicación masivos y hasta de trolls y bots - puestas en circulación en 2016 a raíz de un conflicto entre el actual presidente argentino, Mauricio Macri, y el conductor televisivo Marcelo Tinelli.

El tercer agrupamiento reúne escritos cuya ligazón está dada por la presentación de estudios de corte empírico, aunque sostenidos en andamiajes teóricometodológicos diversos. \#Tarifazo. Medios tradicionales y fusión de agenda en redes sociales es el título con que Ernesto Calvo y Natalia Aruguete exponen sus hallazgos sobre la relación entre los medios masivos de comunicación, los discursos que se publican en Twitter y el establecimiento de agenda. Posicionados en una perspectiva actualizada de la agenda setting y específicamente abocados a estudiar los tuits que circularon como respuesta a la decisión del gobierno de Macri de aumentar las tarifas de los servicios públicos, muestran la conexión existente entre el modelo de fusión de agendas (agenda melding) y la metáfora de las burbujas de filtro (filter bubble) elaborada por Pariser (2017). En tanto, el trabajo de Marcelo Santos, Testimonial tweeting. People's voice (and eyes) on anti-impeachment protests in Brazil, vuelve a hacer foco en el rol que tienen las RSI en los movimientos sociales de protesta. De este modo, el autor despliega los resultados de su investigación sobre el rechazo - por parte de ciudadanos comunes nucleados en Twitter a partir del hashtag \#ForaTemeral proceso que conllevó la destitución de la expresidenta Dilma Rousseff en Brasil. A continuación, con una impronta más sociológica y aplicando técnicas metodológicas de corte cualitativo, se ubica el texto de Santiago Galar y Brenda Focas, ¿Víctimas virtuales? Inseguridad, públicos y redes sociales en Argentina. Al recuperar experiencias que se expresan tanto a través de la plataforma de Facebook como mediante la puesta del cuerpo en la calle, dicho artículo recae 
en una de las temáticas que más interpela a la opinión sobre lo público en nuestras sociedades latinoamericanas actuales: la (in)seguridad. Se indaga, allí, especialmente, en ciertas prácticas ejercidas en las RSI por familiares de víctimas del delito en Argentina, durante la última década. Finalmente, la sección Artículos de InMediaciones de la Comunicación concluye con un escrito de Aníbal Rossi, quien comparte un conjunto de reflexiones - orientadas principalmente a revisar las implicancias de los sistemas algorítmicos en la vida social y a tomar distancia crítica respecto del concepto de burbujas de filtro inaugurado por Pariser (2017) — derivadas de los resultados obtenidos de un experimento empírico.

Todos los artículos mencionados pueden ser leídos a la luz de las consideraciones de John Thompson, quien actualiza sus ya clásicas reflexiones sobrela esfera pública en la entrevista Social Media and the Public Sphere que realizamos especialmente para este volumen. Cuando el autor escribió trabajos de referencia en la temática como, por ejemplo, The Theory of the Public Sphere (1993) o The Media and Modernity (1995), las RSI aún no existían. En esta oportunidad, por tanto, las inquietudes que Thompson responde giran alrededor de este nuevo escenario de lo público y de los modos de participación que se suscitan en su seno. En su lengua materna, el autor se explaya sobre temas vinculados con el rol de las RSI en la configuración actual de la esfera pública, así como su lugar en la visibilidad mediada - concepto acuñado por Thompson en los'90o las consecuencias políticas de los modos de visibilidad actual de lo privado y lo íntimo. Asimismo, también analiza cuáles son hoy las categorías teóricas imprescindibles para abordar la creciente complejidad del sistema de medios, ylos alcances yla pertinencia que continúa teniendo la noción de esfera pública para los estudios sociales contemporáneos. El resultado, creemos, es un intercambio rico en conceptualizaciones y cavilaciones para seguir reflexionando sobre los tópicos aquí abordados.

Por último, el lector encontrará en la sección Reseñas las lecturas de tres obras de publicación reciente sobre la temática general del volumen. En primer lugar, la compilación Titulares, hashtags y videojuegos, coordinada por Eugenia Mitchelstein y Pablo Boczkowski, es reseñada por Adriana Amado, quien destaca el valor simbólico de la publicación - en tanto reúnelas presentaciones del primer congreso del Centro de Estudios sobre Medios y Sociedad (MESO) - y hace un repaso sucinto de cada una de ellas. En segundo lugar, Rubén Biselli presenta su mirada sobre La humanidad aumentada. La administración digital del mundo, de Éric Sadin, libro que intenta leer la "administración algorítmica del mundo" (p. 305), relevando indicios de una mutación antropológica que conlleva sus peligros, necesita de resistencia, pero también de aceptación de los aportes de la digitalización global. Por último, el español Joaquín MarquésPascual cierra esta sección con sus apreciaciones sobre el libro de Marc Argemí, El sentido del rumor. Cuando las redes sociales ganan a las encuestas, en el que 
se analiza cómo las redes sociales participan del debate político y el comportamiento de los usuarios a la luz de los conceptos de rumory Fama (en mayúscula, tal como es planteado por Argemí).

Como puede vislumbrarse en esta breve presentación, el Volumen 13(1) de InMediaciones de la Comunicación reúne un conjunto diverso de escritos, tanto en perspectivas como en procedencias institucional y geográfica de los autores. El resultado se traduce, así, en una pluralidad de miradas académicas sobre la configuración de lo público político en la actualidad, posible, también, gracias al acompañamiento comprometido y generoso de reconocidos pares evaluadores de diversas nacionalidades, cuya laboriosa tarea agradecemos. Sólo resta ahora el encuentro - que, esperamos, sea fructífero- entre textos y lectores.

\section{REFERENCIAS}

Pariser, E. (2017). El filtro burbuja. Cómo la red decide lo que leemos y lo que pensamos. Barcelona: Taurus.

Thompson, J. (1995). The Media and Modernity: A Social Theory of the Media. Stanford: Stanford University Press.

Thompson, J. (1993). The theory of the Public Sphere. Theory, Culture and Society, 10(3), 173-189.

Van Dijck, J.(2016). La cultura dela conectividad. Una historia crítica delas redes sociales. Buenos Aires: Siglo XXI.

Verón, E. (2013) La semiosis social, 2. Ideas, momentos, interpretantes. Buenos Aires: Paidós. 
Ma. Cecilia Reviglio es Doctora en Comunicación Social por la Universidad Nacional de Rosario (UNR), Argentina, e Investigadora del Consejo de Investigadores de la Universidad Nacional de Rosario (CIUNR) y codirectora del Proyecto "Nuevas visibilidades en la cultura digital: esfera pública contemporánea y redes sociales en Internet" (Facultad de Ciencia Política y RRII, UNR). Desarrolla su tarea docente en la Licenciatura en Comunicación Social, la Especialización en Psicología Clínica, Institucional y Comunitaria, la Maestría en Enseñanza de la Lengua y la Literatura, todas de la UNR así como en el Profesorado de Lengua y Literatura del Instituto de Enseñanza Superior "Olga Cossettini" de la provincia de Santa Fe. Es miembro del Comité Académico del Centro de Investigaciones en Mediatizaciones (CIM-UNR). Ha publicado artículos, papers y capítulos de libros en diversas publicaciones especializadas en el área de la comunicación, entre los que se destaca la compilación junto con Natalia Raimondo Anselmino Territorios de comunicación. Recorridos de investigación para abordar un campo heterogéneo (Quito: Ciespal, 2013).

Natalia Raimondo Anselmino es Doctora en Comunicación Social por la Universidad Nacional de Rosario (UNR) e investigadora del Consejo Nacional de Investigaciones Científicas y Técnicas (CONICET), Argentina. Se desempeña como docente adjunta en la Licenciatura en Comunicación Social de la UNR (cátedra Lenguajes III) y en la Licenciatura en Diseño Gráfico de la Universidad Abierta Interamericana (UAI). Dicta, asimismo, cursos de posgrado en la UNR y otras universidades. Es miembro del Comité Académico del Centro de Investigaciones en Mediatizaciones (CIM) e investigadora afiliada al Centro de Estudios sobre Medios y Sociedad (MESO) de Universidad de San Andrés y Northwestern University. Desarrolla tareas de investigación en el campo de la semiótica de los medios, teniendo a la prensa y a las redes sociales en Internet como objetos privilegiados de estudio. Entre sus publicaciones se destacan el libro La prensa online y su público. Un estudio de los espacios de intervención y participación del lector en Clarín y La Nación (Buenos Aires: Teseo, 2012) y la compilación junto con Ma. Cecilia Reviglio Territorios de comunicación. Recorridos de investigación para abordar un campo heterogéneo (Quito: Ciespal, 2013). 